\title{
Scanner based Fingerprint Matching system for Security Applications
}

\author{
Kuljinder Singh ${ }^{1}$, Maninder Kaur ${ }^{2}$ \\ ${ }^{1,2}$ DIET, Kharar, Punjab, India
}

\begin{abstract}
In the presented work, it is proposed to enhance the finger print image quality based on an adaptive fingerprint enhancement method that is based on contextual filtering. The term adaptive implies that parameters of the method are automatically adjusted based on the input fingerprint image. Once the finger print image is enhanced at the required level, the pores are extracted based on segmentation of the finger print image by eliminating the finger print area above a threshold limit. The pores location and then interdistances are computed and stored in a data base. Further, the minutiae are extracted and again their location and inter-distances are computed and stored in a data base. Same procedure is repeated for the query image and standard deviation is computed between the inter-distance of the query image and data base images. The finger print information based on pores and minutiae are fused together in order to get the matching score.
\end{abstract}

Keywords: Scanner based Fingerprint Matching system for Security Applications

\section{Introduction}

A fingerprint is the feature pattern of one finger. As each and every person has unique and permanent fingerprint so fingerprints have being used for identification and forensic investigation. The fingerprint recognition is the oldest and most reliable biometric used for personal identification. The ancient procedure of matching fingerprints used fingerprint sensor technology which was extremely costly and timeconsuming. Need is to develop a system which is easy to operate and is cost effective.

The proposed work is to develop a document scanner and machine vision based finger print matching system which will be cost effective, fast and reliable. Fingerprint classification system need to extract required significant features from the image and match against the stored features of fingerprint samples of the database. This further requires image of fingerprint in the form of the inked based impression and matching of the fingerprint by the developed algorithm.

Jing bao Sweat pores have been recently employed for automated fingerprint recognition, in which the pores are usually extracted by using a computationally expensive skeletonization method. The fingerprint image is partitioned into blocks and a local pore model is determined for each block [1].

Qijun Zhao Sweat pores on fingerprints have proven to be discriminative features and have recently been successfully employed in automatic fingerprint recognition systems (AFRS), where the extraction of fingerprint pores is a critical step. Most of the existing pore extraction methods detect pores by using a static isotropic pore model; however, their detection accuracy is not satisfactory due to the limited approximation capability of static isotropic models to various types of pores [2].

Fingerprint friction ridge details are generally described in a hierarchical order at three different levels, namely, Level 1 (pattern), Level 2 (minutia points), and Level 3 (pores and ridge contours). Although latent print examiners frequently take advantage of Level 3 features to assist in identification, Automated Fingerprint Identification Systems (AFIS) currently rely only on Level 1 and Level 2 features [3].

The circular ROI is divided into non-overlapping blocks. After decomposing the ROI by using DFB, the seven $\mathrm{Hu}$ invariant moments were computed from each block of ROI as the feature of fingerprint features. The proposed scheme can improve performance of verification and is more robust with respect to the fingerprint image quality [4].

Fingerprint identification is accomplished at three levels: pattern, minutia and pores. Nowadays, the Automated Fingerprint Identification Systems (AFIS) is mainly based in pattern types and minutia from information acquire in 500 dpi. Nevertheless, two years ago the National Institute of Standards and Technology (NIST has approved inclusion of the third level, pores based identification and recommend the use of 1000 dpi fingerprint images [5].

High-resolution automated fingerprint recognition systems (AFRSs) offer higher security because they are able to make use of level-3 features, such as pores, that are not available in lower resolution (< 500-dpi) images. One of the main parameters affecting the quality of a digital fingerprint image and issues such as cost, interoperability, and performance of an AFRS is the choice of image resolution [6].

Poor-quality images mostly result in spurious or missing features, which further degrade the overall performance of the recognition systems. This work augments the fingerprint quality with respect to one of the level 3 micro features, i.e., sweat pores [7].

Fingerprint based security systems are implemented for secure access at various levels. Liveness detection is an emerging challenge in the field of fingerprint biometrics 8] .

The performance of Automatic Fingerprint Identification Systems (AFIS) relies on the quality of the input fingerprints, so the enhancement of noisy images is a critical step [9]. 


\section{International Journal of Science and Research (IJSR) \\ ISSN (Online): 2319-7064}

Index Copernicus Value (2013): 6.14 | Impact Factor (2015): 6.391

A key step of the AFIS (Automatic Fingerprint Identification System) is the fingerprint image enhancement. In this paper, an algorithm based on elliptical Gabor filter is presented. The method can determine the range of filtering dynamically by collecting the ridge information on the fingerprint [10].

Automatic Fingerprint Recognition Systems (AFRSs) rely on minutiae position and orientation within the fingerprint image for matching. Minutiae information is highly accurate provided that the fingerprint image matched is of high quality [11].

\section{Methodology}

The proposed system consists of the following steps:

- Finger Print Image Acquisition

- RGB to Gray Scale Conversion

- Image Enhancement:

- Image Binarization

- Pores Extraction

- Minutia Extraction

- GLCM Extraction

- Contrast, Entropy, Energy, Homogeneity, Power and Correlation Computation

- Data base of feature

- Finger print matching

\section{Image Acquisition and Preprocessing}

Finger print images are acquired using the finger print scanner. The acquired image is in jpeg format and is read in matlab using the command imread(). The image is now converted to gray image using rgb2gray() function. The gray image is enhanced using the histogram equalization algorithm. Following figure show the result of image preprocessing operations:

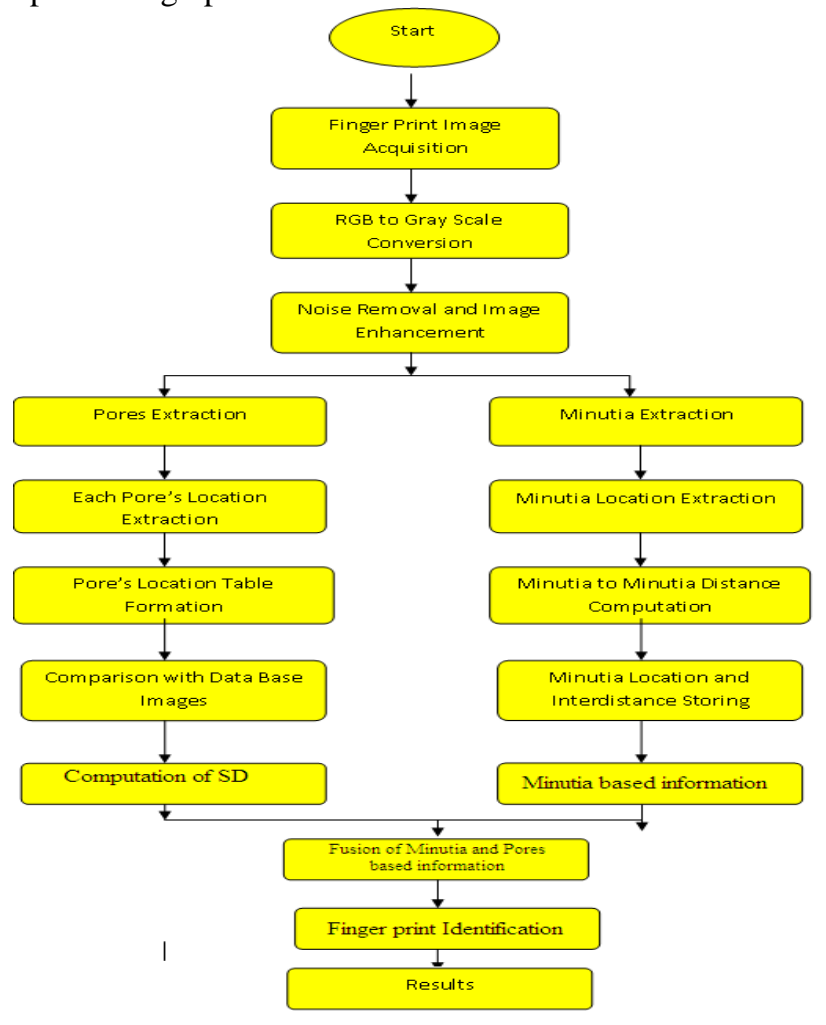

\section{Pores Extraction}

Pores are extracted using the segmentation based on pixel neighborhood connectivity. After image enhancement, the fingerprint image is binarized using otsu algorithm. Further, the image is segmented by removing the connected regions of pixel groups more than 10 . Now, the image is left with only the pores. The pore location is extracted by computing the centre of mass using 1 st order moments. The pore locations are stored in an array for further processing.

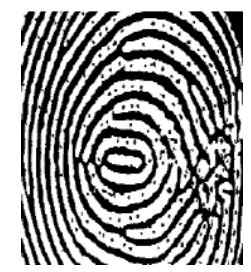

Figure 3

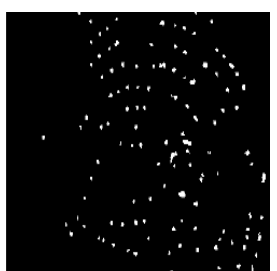

Figure 4

\section{Minutiae Extraction}

Ridge Thinning is to eliminate the redundant pixels of ridges till the ridges are just one pixel wide. The thinned ridge map is then filtered by other three Morphological operations to remove some $\mathrm{H}$ breaks, isolated points and spikes.

After the fingerprint ridge thinning, marking minutia points is relatively easy. But it is still not a trivial task as most literatures declared because at least one special case evokes my caution during the minutia marking stage. In general, for each $3 \times 3$ window, if the central pixel is 1 and has exactly 3 one-value neighbors, then the central pixel is a ridge branch. If the central pixel is 1 and has only 1 one-value neighbor, then the central pixel is a ridge ending.

Together with the minutia marking, all thinned ridges in the fingerprint image are labeled with a unique ID for further operation. The labeling operation is realized by using the Morphological operation: BWLABEL

\section{Fusion of Texture Props, Finger Print Pore Information and Minutia Information}

Pore to pore distance is computed using the following distance formula:

$$
\mathrm{D}=\sqrt{ }(\mathrm{x} 1-\mathrm{x} 2) 2+(\mathrm{y} 1-\mathrm{y} 2) 2
$$

Where $(\mathrm{x} 1, \mathrm{y} 1)$ and $(\mathrm{x} 2, \mathrm{y} 2)$ are the pore's coordinates/locations.

Similarly minutia location and minutia to minutia distance is computed using above formula for query image as well as the data base images. 


\section{International Journal of Science and Research (IJSR) \\ ISSN (Online): 2319-7064 \\ Index Copernicus Value (2013): 6.14 | Impact Factor (2015): 6.391}

The finger print matching score is computed based on the fusion of pore's and minutia information using the following formula:

$$
\text { Score }=\alpha * \operatorname{ED}(\text { Pore })+\beta * \operatorname{ED}(\text { Minutia })
$$

Where ED is the Euclidean distance between the pores and minutia distance vectors. As the resolution increases, the constant $\alpha$ increases and $\beta$ decreases and vice versa. This is on the fact that as the resolution increases or improves, the pores becomes significant and minutia count reduces and the problem becomes finger print matching based on pores. And as the resolution decreases, the pores becomes less significant and minutia count increases and the problem becomes minutia based finger print matching. Therefore, there is a trade off between the resolution and the finger print matching based on pores and minutia.

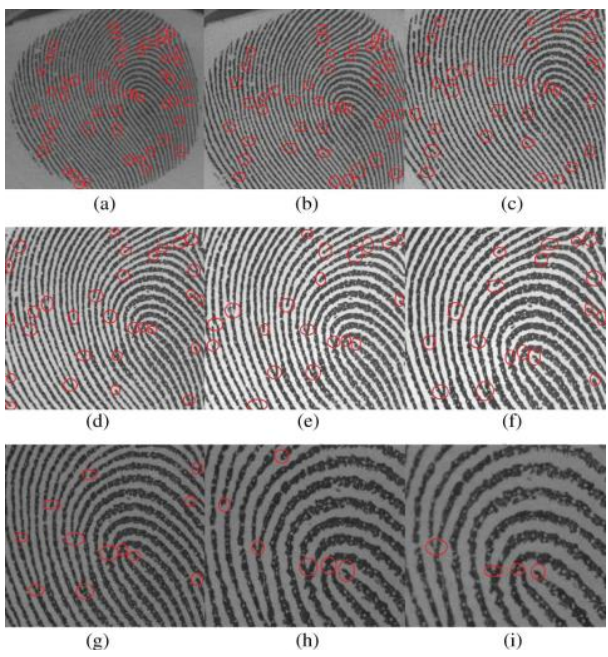

\section{Results}

Two indexes are well accepted to determine the performance of a fingerprint recognition system: one is FRR (false rejection rate) and the other is FAR (false acceptance rate). For an image database, each sample is matched against the remaining samples of the same finger to compute the False Rejection Rate. If the matching $g$ against $h$ is performed, the symmetric one (i.e., h against $\mathrm{g}$ ) is not executed to avoid correlation. All the scores for such matches are composed into a series of Correct Score. Also the first sample of each finger in the database is matched against the first sample of the remaining fingers to compute the False Acceptance Rate. If the matching $\mathrm{g}$ against $\mathrm{h}$ is performed, the symmetric one (i.e., h against $g$ ) is not executed to avoid correlation. All the scores from such matches are composed into a series of Incorrect Score.

In the proposed work, a pore based finger print identification system is proposed. The proposed system works better in comparison to minutia based as the pore density is much higher than the minutia. It may be observed that pore to minutia density may be in the ratio of 10:1. i.e. for one minutia, there are ten no.pf pores in a finger print image. In the presented approach, pore to pore distance network has been generated and that has been used for finger print based person identification. As more complex is the distance fabric network, more it is difficult to break the identity.
Also, the minutia based finger print identification again uses the inter minutia distances. But, as the minutia based distances are dependent upon the no. of minutiae. The pore based finger print identification gives more repeatable results for the same finger matching even if the query finger print is cut from any side or any partial finger print is provided for its match from the data base.

\section{Conclusion}

In the presented work, it has been observed that the finger print identification based on minutia and pores at different resolutions gives a break even, beyond which either of the two method survives. It has observed that at higher resolution, pores based finger print identification gives a better results as the pores become more significant as compared to minutia or it can be said that the minutia count reduces. The accuracy of the finger print identification depends upon how much minutia or pores are available for feature extraction.

Similarly, as the resolution is decreased, minutia becomes significant in numbers and pores become invisible. At that time, accuracy due to minutia is better. However, when the resolution is varied over a range, there is point at which accuracy becomes almost equal based on pores and minutia. This may be observed from the graphical representation of the results given in result section.

The complete algorithm has been developed in matlab software. A finger print data base of more than 100 persons is generated using the Futronic make finger print scanner with variable resolution adjustments.

\section{References}

[1] Qijun Zhao" Adaptive fingerprint pore modeling and extraction",2010

[2] Lin Hong. "Automatic Personal Identification Using Fingerprints", Ph.D. Thesis, 1998.

[3] D.Maio and D. Maltoni. Direct gray-scale minutiae detection in fingerprints. IEEE Trans. Pattern Anal. And Machine Intell., 19(1):27-40, 1997.

[4] Jain, A.K., Hong, L., and Bolle, R.(1997), "On-Line Fingerprint Verification,” IEEE Trans. On Pattern Anal and Machine Intell, 19(4), pp. 302-314.

[5] N. Ratha, S. Chen and A.K. Jain, "Adaptive Flow Orientation Based Feature Extraction in Fingerprint Images", Pattern Recognition, Vol. 28, pp. 1657-1672, November 1995.

[6] Alessandro Farina, Zsolt M.Kovacs-Vajna, Alberto leone, Fingerprint minutiae extraction from skeletonized binary images, Pattern Recognition, Vol.32, No.4, pp877-889, 1999.

[7] Lee, C.J., and Wang, S.D.: Fingerprint feature extration using Gabor filters, Electron. Lett., 1999, 35, (4), pp.288-290.

[8] M. Tico, P.Kuosmanen and J.Saarinen. Wavelet domain features for fingerprint recognition, Electroni. Lett., 2001, 37, (1), pp.21-22.

[9] L. Hong, Y. Wan and A.K. Jain, "Fingerprint Image Enhancement: Algorithms and Performance Evaluation", 


\section{International Journal of Science and Research (IJSR) \\ ISSN (Online): 2319-7064}

Index Copernicus Value (2013): 6.14 | Impact Factor (2015): 6.391

IEEE Transactions on PAMI ,Vol. 20, No. 8, pp.777789, August 1998.

[10] Image Systems Engineering Program, Stanford
University.
Student
project
By

ThomasYeo,WeePengTay,YingYuTai.

Volume 5 Issue 6, June 2016

www.ijsr.net

Licensed Under Creative Commons Attribution CC BY 